ORIGINAL ARTICLE

\title{
Healthcare use before and after a workplace injury in British Columbia, Canada
}

\author{
J A Brown, P McDonough, C A Mustard, H S Shannon

See end of article for authors' affiliations .....................

Correspondence to: Ms J A Brown, Department of Occupational Health and Environmental Medicine, McMaster University, HSC $3 \mathrm{H} 57$ Hamilton, ON, Canada; judy.brown@utoronto.ca

Accepted 10 February 2006 Published Online First 23 February 2006
Objectives: There is growing evidence that occupational injuries influence workers' emotional and physical wellbeing, extending healthcare use beyond what is covered by the Workers' Compensation Board (WCB).

Methods: The authors used an administrative database that links individual publicly funded healthcare and WCB data for the population of British Columbia (BC), Canada. They examined change in service use, relative to one year before the injury, for workers who required time off for their injuries (lost time $=\mathrm{LT}$ ) and compared them to other injured workers (no lost time $=\mathrm{NLT}$ ) and individuals in the population who were not injured (non-injured $=\mathrm{NI})$.

Results: LT workers increased physician visits (22\%), hospital days (50\%), and mental healthcare use $(43 \%$ physician visits; and $70 \%$ hospital days) five years after the injury, relative to the year before the injury, at a higher rate than the $\mathrm{NI}$ group. For the NLT workers, the level of increased use following the injury was between that of these two groups. These patterns persisted when adjusting for registration in the $\mathrm{BC}$ Medical Service Plan (MSP) and several workplace characteristics.

Conclusions: Although the WCB system is the primary mechanism for processing claims and providing information about workplace injury, it is clear that the consequences of workplace injury extend beyond what is covered by the WCB into the publicly funded healthcare system. l: Canada, in 2003, there were approximately 800000 workplace injuries and illnesses reported by the Association of Workers' Compensation Boards of Canada (AWCBC), representing approximately one in 16 Canadians. ${ }^{1}$ In the United States (US) the figures are similar with approximately 4.5 million occupational injuries reported in 2003, representing approximately one in 20 full time workers. $^{2}$ Approximately $50 \%$ of these injuries resulted in disabilities requiring recuperation away from work or restricted activities during work hours. ${ }^{2}$ While only a small proportion of work injuries result in substantial disability and a lengthy time away from work, these injuries have the greatest emotional, social, and economic consequences.

The National Occupational Research Agenda (NORA), established by the National Institute for Occupational Safety and Health (NIOSH), named "social and economic consequences of workplace illness and injury" as one of the 21 priorities for the 21 st century. ${ }^{3}$ Recent research in the area has documented, among other things, the reduced earnings, long term physical limitations, and mental health problems experienced by workers as a result of their injury (for example, $\left.\mathrm{see}^{4-8}\right)$. However, most are primarily based on qualitative observations, rather than systematic population based studies and thus, cannot suggest the extent to which these consequences can be generalised to all injured workers. Given the growing evidence that occupational injuries influence workers' emotional and physical wellbeing, and that these consequences extend beyond the workers' compensation system into the social and economic systems of society, there is a need to determine the extent of these problems on a population level.

Higher rates of post-injury use of healthcare services are likely to be indicative of ongoing problems related to the injury and, hence, serve as an indirect measure of the above mentioned consequences of injury. Our study examined the overall healthcare and mental healthcare use of workers who filed a claim to the Workers' Compensation Board (WCB) in
1994 and missed days of work due to the injury. We examined Medical Service Plan (MSP) and hospital discharge records, contained in the British Columbia Linked Health Dataset (BCLHD), for workers five years before and five years after the injury. We compared their utilisation to injured workers who did not require time off for their injury and individuals in the population who did not file a claim between 1989 and 1999 .

\section{METHODS}

\section{The BCLHD}

The BCLHD links individual MSP payment data, hospital separations, and WCB data for the entire population of British Columbia (BC), Canada.

In $\mathrm{BC}$, the workers' compensation system is a publicly administered single payer system, funded exclusively by payroll levies on industry. It covers over $90 \%$ of workers in the province, and is the single source of wage loss compensation for a workplace injury. Approximately $95 \%$ of the transaction records in each file are linked to an individual recorded on the Registration and Premium Billing file (R\&PB) maintained by the MSP. ${ }^{9}$ In BC, the MSP insures medically required services provided by physicians, other healthcare professionals, laboratory services, and diagnostic procedures. Under the Medicare Protection Act, enrolment with MSP is mandatory for all residents. Coverage is usually available through the employer or is self-administered. When leaving and entering the province, residents are required to notify the MSP of the date as soon as possible or risk having no coverage or being billed for services they no longer need.

In BC, MSP numbers are part of the employment arrangement; employers pay for employees' medical

Abbreviations: BC, British Columbia; BCLHD, British Columbia Linked Health Dataset; LT, lost time injured workers; MSP, Medical Service Plan; $\mathrm{NI}$, non-injured comparisons; NLT, no lost time injured workers; WCB, Workers' Compensation Board. 
premiums and benefits, which result in the assignment of a MSP number. The employee is given 00 as the final two digits of their MSP number, their spouse a 01 designation, all dependents a $02,03,04 \ldots$ designation (except for newborns who are given a 66 designation). Thus, we were able to look at injured workers who were 00 (and thus were registered as the principal employee) during the entire study period (198999), and their comparisons that were also 00 during the entire study period.

\section{Study subjects}

Information on whether the claim was short term disability (STD, under six months off work), long term disability (LTD, six or more months off work), rejected (REJ), healthcare only (HCO), or fatality (FLT) was provided by the Centre for Health Services and Policy Research (CHSPR) in BC. ${ }^{10}$ Because some workers had more than one claim in 1994, the injury of interest was chosen in the order of most serious claim (FLT, LTD, STD, HCO, and REJ).

All injured workers aged 25 and over who filed a WCB claim in 1994 and lost one or more days of work due to the injury (lost time workers; hereby referred to as LT) were included (that is, STD or LTD).

A comparison group was sampled from the MSP, consisting of individuals in the general population who were registered for health benefits but did not submit a WCB claim between 1989 and 1999 (non-injured; hereby referred to as NI). They were individually matched to the LT injured workers on sex, age, and income decile (l (lowest) to 10 (highest)) of residential neighbourhood (hereafter referred to as income decile).

A second comparison group consisted of workers who filed a WCB claim in 1994 but did not require time off for their injuries (HCO or REJ, no lost time; hereby referred to as NLT). Workers who died as the result of their injuries (FTL) were not included.

\section{Measurement}

MSP and hospital discharge records from 1989 to 1999 were reviewed (five years before and five years after the date of the 1994 injury).

To assess the possible effect of including workers with other claims before or after the 1994 injury, who may have chronic conditions that require long term physician care, we analysed the data comparing workers with one claim, to workers with repeat claims between 1989 and 1999 (before 1994 but not after, after 1994 but not before, and both before and after 1994). Using coding from the Canadian Work Injuries Standard (CWIS), injury types were separated into "traumatic"(for example, fractures, cuts, lacerations) and "chronic" (for example, low back pain, repetitive strain, hearing loss) to detect possible differences in patterns of use by disability. ${ }^{11}$ The groups were also examined according to the length of time off work (more than 12 weeks and less than 12 weeks).

As the NI group was not injured between 1989 and 1999, it is possible that many are not in the workforce and thus do not have the potential to be injured (and thus are not comparable). Therefore we also looked at individuals that were in the workforce the entire study period (1989-99).

To assess the possibility that individuals who remain in the system use healthcare services differently from individuals who come and go, another analysis was restricted to individuals registered in the BC MSP for the entire study period.

General practitioner visits were determined using project case numbers, date of service, and specialty code. The International Classification of Diseases, Ninth Revision (ICD-9) in the Diagnostic and Statistical Manual of Mental
Disorders, 4th edition (DSM-IV) was used to assess service use for overall mental healthcare (290-319) and depression (296.1-296.7, 296.9, 300.4, and 311). All physician (general practitioners (GPs) and specialists) service use was assessed for mental healthcare. Specific ICD-9 codes before 1992 were not available in the MSP data. To assess differences in severity, mental healthcare use was divided into psychotic, non-psychotic, and other disorders based on previous research classifying mental health disorders. ${ }^{12}{ }^{13}$ WCB funded physician services are included in the MSP data sets.

Days of hospitalisation for overall and mental healthcare use were determined from the Hospital Admissions and Separations (HAS) data. The HAS data included ICD-9 codes back to 1989. WCB funded services were not included in the hospital data.

To assess the impact of WCB related mental illness, the data were re-analysed leaving out workers who had filed a mental illness claim between 1989 and 1999.

\section{Data analysis}

To account for any differences between the injured worker groups and the NI in registration for MSP benefits, a yearly weighting variable was created (from 0 to $100 \%$ ), to account for any differences between the workers and the comparisons in registration time for MSP benefits. Thus, if an individual was not registered with the MSP programme during the yearly period of interest, they were not included in the denominator. The fact that some individuals were registered for only a proportion of the year was accounted for by the MSP registration weighting variable.

Measures of healthcare use were calculated for each 12 month period, five years before and five years after the 1994 injury (yearly periods 5th to 1st pre-injury and yearly periods 1 st to 5 th post-injury). The first yearly period after the injury began on the date of injury.

We estimated yearly percentage change in mean visits before and after the injury, relative to the mean one year before the injury for each group. We also present the percentage of individuals who increase use, decrease use, and remain the same one year and five years after the injury.

As we examined an entire population of injured workers and have an extremely large population, we have not provided estimates of sample variation for overall GP visits and days in hospital. For mental healthcare use (given the smaller numbers) we present results using repeated measures analysis of variance (ANOVA), with group and time as the factors of interest and their interaction showing an effect of the injury. As the same results were achieved when we ran a mixed model, for clarity, we decided to present the ANOVA. Proportions were compared with the $\chi^{2}$ test.

\section{RESULTS}

There were 52319 LT (and 52319 matched NI) and 69142 NLT workers eligible for the study. Seventy three per cent of the LT injured workers and $74 \%$ of the NLT workers were male. At the time of injury, the mean age of LT injured workers was 39.9 years (SD 10.6) and the mean income decile was 4.9 (SD 2.7, range 1-10); means for NLT injured workers were 39.6 years (SD 10.2) and 5.3 (SD 2.8), respectively.

\section{Registration in the BC MSP}

Throughout the study period, both groups of injured workers were more likely than the NI group to be registered in the BC MSP. Five years before the injury, $77 \%$ of LT and $81 \%$ of NLT injured workers were registered to receive services, compared to $61 \%$ of the NI. One year pre-injury, $89 \%$ of LT and $92 \%$ of NLT injured worker were registered, compared to $86 \%$ of the 


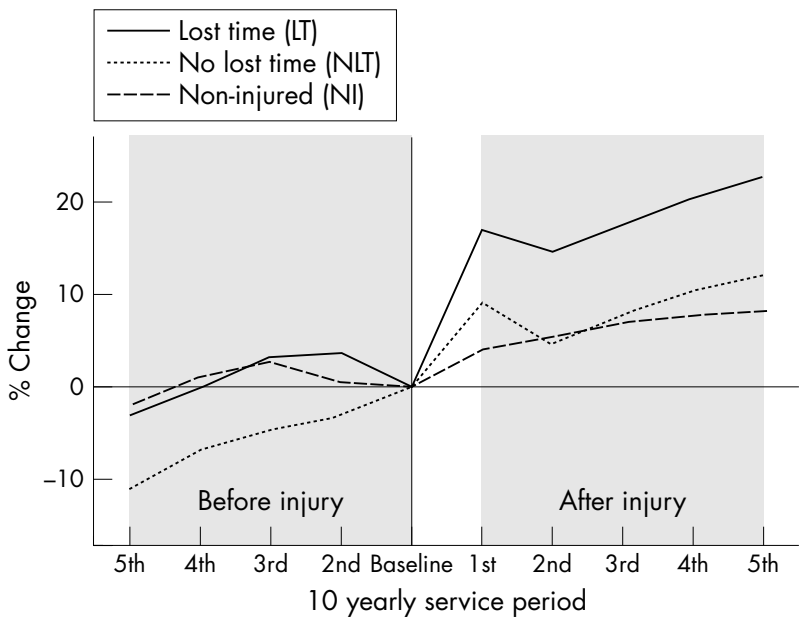

Figure 1 General practitioner use of injured workers five years before and five years after a 1994 workplace injury. The baseline period (vertical line) is the first yearly period immediately before the injury (see table 1). Data for other periods show percentage differences from these means.

NI. By the end of the study period (five years after) $89 \%$ of LT and $91 \%$ of NLT workers were registered, compared to $83 \%$ of the NI.

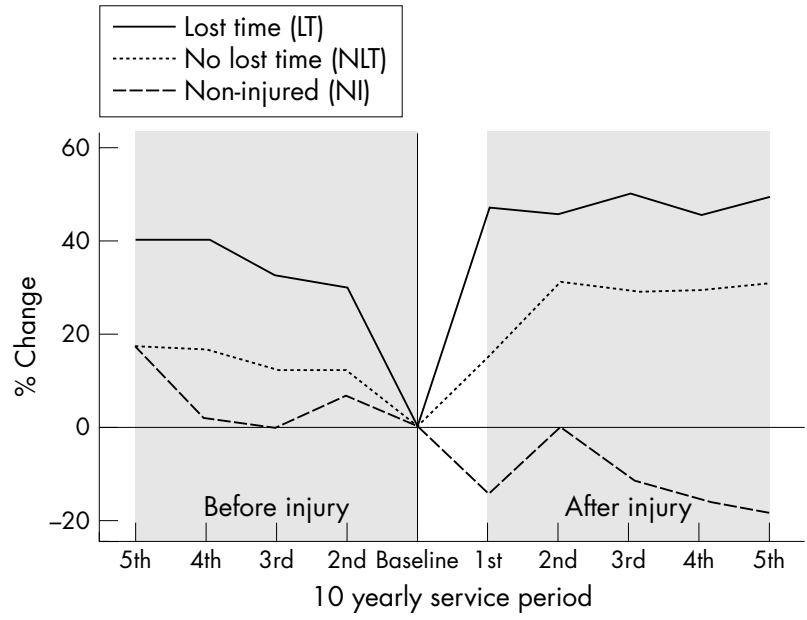

Figure 2 Hospital use of injured workers five years before and five years after a 1994 workplace injury. The baseline period (vertical line) is the first yearly period immediately before the injury (see table 2). Data for other periods show percentage differences from these means.

\section{General practitioner use}

Fifty two per cent of the LT increased GP visits one year after the injury compared to $47 \%$ of the NLT and $40 \%$ of the NI.

Table 1 Percentage change in mean general practitioner visits following a workplace injury relative to baseline (one year before injury), by claim and registration characteristics

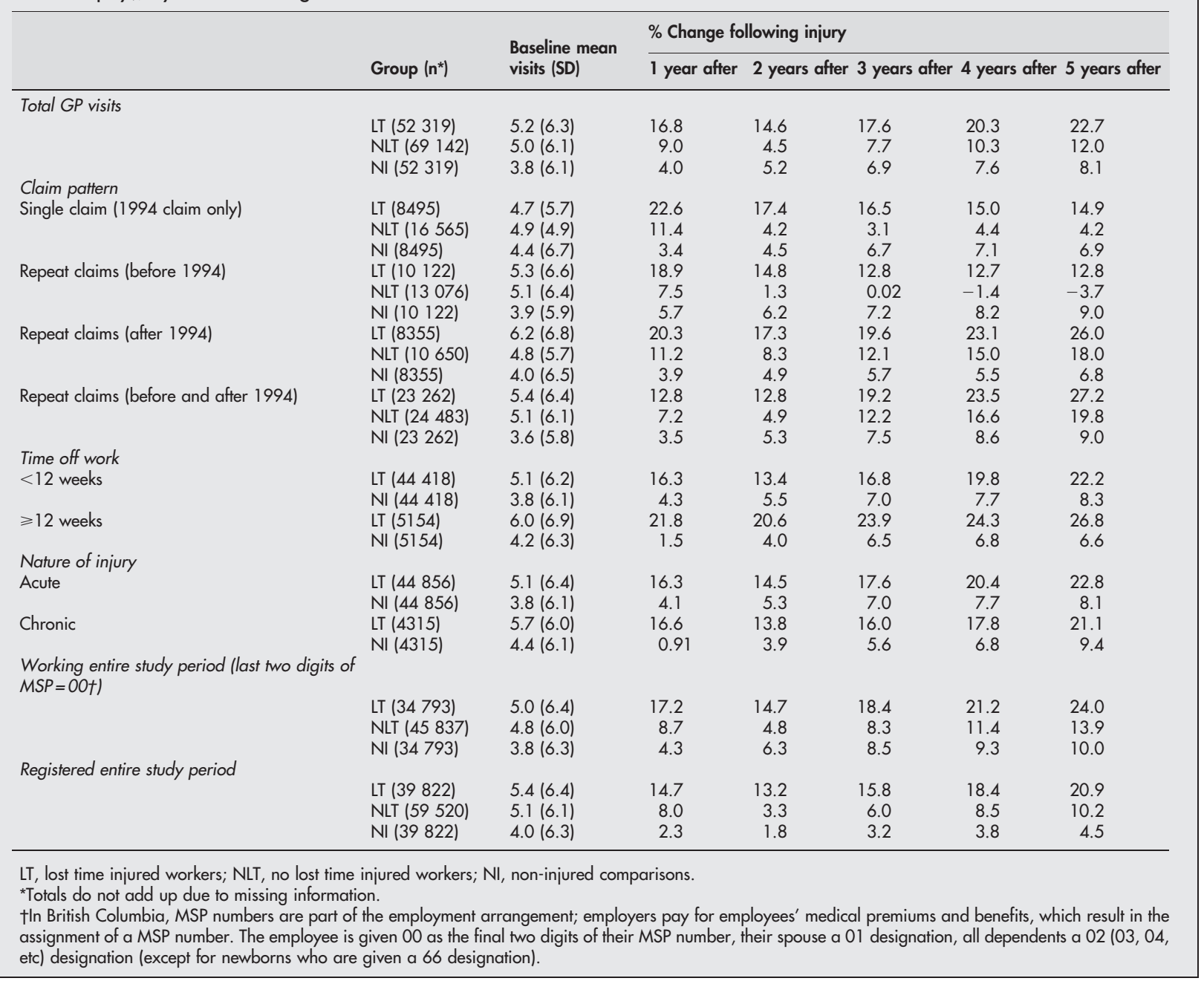


Five years after the injury, 52\% of LT had an increase in use, compared with $47 \%$ of the NLT and $42 \%$ of the NI (data not shown).

Figure 1 shows the percentage change in mean GP visits before and after the injury, relative to each group's mean in the year before the injury. The LT workers increased use by $16 \%$ one year after the injury, dropped somewhat, and then increased to $22 \%$ by the end of the study period. In contrast, the NI experienced a slow and constant increase to $8 \%$ in visits in the final year under study. The NLT workers increased by $12 \%$ by the end of the study period. Service use before the injury was relatively stable for the LT injured workers and NI and increased somewhat for the NLT workers.

Table 1 shows the relative percentage change in mean GP visits following a workplace injury by worker claim and registration characteristics. LT repeat claimants (after 1994 only and before and after 1994) had higher increases compared to LTs with one claim or repeat claims before 1994. However, even among single claimants the pattern of increase for the LTs was greater than the other two groups. LT workers who were off more than 12 weeks had a higher percentage increase in use compared to LTs who had less than 12 weeks off work. Acute LT workers had a slightly higher percentage increase in use compared to LTs with chronic injuries. In both cases, LTs had the greatest percentage increase compared to the NIs (see table 1).

When we look at individuals who were working the entire study period (last two digits of MSP number $=00$ ) and workers who were registered the entire period of the study, the pattern of LTs having the greatest increase in service use and the NIs having the smallest increase in use, remains the same (see table 1).

\section{Hospital use}

Seven per cent of the LT workers increased hospital days one year after the injury, compared to $5 \%$ of the NLT workers and $4 \%$ of the NI workers. Five years after the injury, $6 \%$ of LTs had an increase in days, compared to $5 \%$ of the NLTs and $4 \%$ of the NIs (data not shown).

The NI group were less likely to have a hospital admission but once there, they spent more days on average. Among those with inpatient hospital stays, $23 \%$ of the NIs spent 10 or more days in hospital in the first year after the injury, compared to $15 \%$ of LT workers and $14 \%$ of NLT workers $\left(\chi^{2}=108.2, \mathrm{df}=2, \mathrm{p} \leqslant 0.001\right)$. Both groups of injured workers had higher mean days in hospital compared to the year before the injury (data not shown).

Figure 2 shows the percentage change in mean days in hospital before and after the injury, relative to each group's mean in the year before the injury. The LT injured workers increased their use in the year after the injury by $7 \%$; use then levelled off to a $50 \%$ increase in days by the end of the study period. The NLT workers had a similar (but smoother) pattern albeit with smaller increases, up to $30 \%$ more days by the end of the study period. In contrast, the NI had little change, with a decrease (15\%) in the first year post-injury,

Table 2 Percentage change in mean hospital days following a workplace injury relative to baseline (one year before injury), by claim and registration characteristics

\begin{tabular}{|c|c|c|c|c|c|c|c|}
\hline & \multirow[b]{2}{*}{ Group $\left(n^{*}\right)$} & \multirow{2}{*}{$\begin{array}{l}\text { Baseline mean } \\
\text { days (SD) }\end{array}$} & \multicolumn{5}{|c|}{$\%$ Change following injury } \\
\hline & & & 1 year after & 2 years aft & 3 years 0 & 4 years & 5 years after \\
\hline \multicolumn{8}{|l|}{ Total hospital days } \\
\hline & LT (52 319) & 0.24 (1.9) & 7.0 & 40.6 & 50.3 & 45.5 & 49.7 \\
\hline & NLT (69 142) & $031(3.5)$ & 15.5 & 31.2 & 29.4 & 29.6 & 30.8 \\
\hline & NI (52 319) & $0.64(10.6)$ & -14.6 & 03 & -11.2 & -15.7 & -18.6 \\
\hline \multicolumn{8}{|l|}{ Claim pattern } \\
\hline \multirow[t]{3}{*}{ Single claim (1994 claim only) } & LT (8495) & $0.31(2.7)$ & 59.5 & 50.8 & 49.3 & 47.1 & 47.4 \\
\hline & NLT (16 565) & $0.45(5.8)$ & 21.4 & 43.3 & 30.4 & 20.0 & 25.0 \\
\hline & $\mathrm{NI}(8495)$ & $0.86(13.0)$ & -35.7 & -6.7 & -0.88 & -8.2 & -26.0 \\
\hline \multirow[t]{3}{*}{ Repeat claims (before 1994) } & LT (l $\left.\begin{array}{ll}10 & 122\end{array}\right)$ & $0.28(2.4)$ & 57.8 & 58.6 & 60.3 & 54.0 & 59.8 \\
\hline & NLT (13 076) & $0.33(2.7)$ & 24.6 & 31.4 & 35.2 & 42.4 & 27.8 \\
\hline & $\mathrm{NI}\left(\begin{array}{lll}10 & 122\end{array}\right)$ & $0.73(14.1)$ & -4.6 & -1.8 & -32.9 & -45.7 & -13.7 \\
\hline \multirow[t]{3}{*}{ Repeat claims (after 1994) } & LT (8355) & $0.20(1.4)$ & 42.2 & 46.5 & 46.3 & 40.3 & 51.5 \\
\hline & NLT (10 650) & 0.25 (1.9) & -2.8 & 18.6 & 21.7 & 13.4 & 42.1 \\
\hline & $\mathrm{NI}(8355)$ & $0.67(7.2)$ & -38.0 & -31.4 & -35.0 & -27.4 & -23.4 \\
\hline \multirow[t]{3}{*}{ Repeat claims (before and after 1994) } & LT (23 262) & $0.21(1.5)$ & 24.8 & 28.4 & 44.7 & 40.7 & 42.8 \\
\hline & NLT (24 483) & $0.23(2.0)$ & 3.8 & 14.3 & 27.5 & 35.3 & 34.4 \\
\hline & $\mathrm{NI}(23262)$ & $0.51(8.7)$ & -2.2 & 15.0 & 1.3 & -2.1 & -14.9 \\
\hline \multicolumn{8}{|l|}{ Time off work } \\
\hline \multirow[t]{2}{*}{$<12$ weeks } & LT (44 418) & $0.22(1.8)$ & 44.7 & 46.7 & 50.0 & 46.2 & 50.3 \\
\hline & NI $(44418)$ & $0.63(11.1)$ & -19.7 & -8.7 & -24.3 & -25.6 & -25.9 \\
\hline \multirow[t]{2}{*}{$\geqslant 12$ weeks } & LT (5154) & $0.28(1.6)$ & 62.3 & 51.8 & 60.9 & 50.1 & 58.8 \\
\hline & NI (5154) & $0.57(5.0)$ & 9.1 & 34.9 & 15.1 & -5.9 & 15.9 \\
\hline \multicolumn{8}{|l|}{ Nature of injury } \\
\hline \multirow[t]{2}{*}{ Acute } & LT (44 856) & $0.22(1.8)$ & 48.2 & 46.5 & 51.3 & 47.0 & 52.0 \\
\hline & $\mathrm{NI}(44856)$ & $0.64(11.0)$ & -0.42 & 38.2 & 44.7 & 31.5 & 12.4 \\
\hline \multirow[t]{2}{*}{ Chronic } & LT (4315) & $0.39(4.7)$ & 26.1 & 34.5 & 39.2 & 36.5 & 28.0 \\
\hline & NI (4315) & $0.76(6.8)$ & -0.42 & 38.2 & 44.7 & 31.5 & 12.4 \\
\hline \multicolumn{8}{|c|}{$\begin{array}{l}\text { Working entire study period (last two digits } \\
\text { of } M S P=00+\text { ) }\end{array}$} \\
\hline & LT (34 793) & $0.23(2.0)$ & 47.0 & 45.9 & 53.0 & 47.5 & 50.6 \\
\hline & NLT (45 837) & $0.33(4.0)$ & 9.0 & 31.1 & 28.0 & 28.7 & 31.3 \\
\hline & NI (34 793) & 0.77 (12.7) & -27.0 & -0.41 & -13.9 & -18.9 & -27.8 \\
\hline \multicolumn{8}{|l|}{ Registered entire study period } \\
\hline & LT (39 822) & 0.24 (1.9) & 45.9 & 44.9 & 48.6 & 44.0 & 48.0 \\
\hline & NLT (59 520) & $0.32(3.6)$ & 10.5 & 30.0 & 24.9 & 25.1 & 26.2 \\
\hline & $\mathrm{NI}(39$ 822) & $0.65(11.0)$ & -12.8 & 0.33 & -15.2 & -20.1 & -24.0 \\
\hline
\end{tabular}

LT, lost time injured workers; NLT, no lost time injured workers; NI, non-injured comparisons.

*Totals do not add up due to missing information.

†See table 1 footnotes for details. 
Table 3 Percentage change in mean physician visits for mental healthcare following a workplace injury relative to baseline (one year before injury), by claim and registration characteristics

\begin{tabular}{|c|c|c|c|c|c|c|c|}
\hline & \multirow[b]{2}{*}{ Group $\left(n^{*}\right)$} & \multirow{2}{*}{$\begin{array}{l}\text { Baseline mean } \\
\text { visits (SD) }\end{array}$} & \multicolumn{5}{|c|}{$\%$ Change following injury } \\
\hline & & & 1 year after & 2 years after & 3 years after & 4 years after & 5 years after \\
\hline \multicolumn{8}{|l|}{ Total physician visits } \\
\hline & LT (52 319) & $0.42(2.6)$ & 19.7 & 29.5 & 33.4 & 37.9 & 42.5 \\
\hline & NLT (69 142) & $0.41(2.4)$ & 15.8 & 22.2 & 26.9 & 30.9 & 35.6 \\
\hline & NI (52 319) & $0.49(3.5)$ & 8.6 & 12.9 & 16.5 & 23.8 & 24.3 \\
\hline \multicolumn{8}{|l|}{ Claim pattern } \\
\hline \multirow[t]{3}{*}{ Single claim (1994 claim only) } & LT (8495) & $0.36(2.0)$ & 26.4 & 35.4 & 38.8 & 39.8 & 48.4 \\
\hline & NLT (16 565) & $0.44(2.7)$ & 18.8 & 20.2 & 22.1 & 28.1 & 32.4 \\
\hline & NI (8495) & $0.57(3.9)$ & 4.1 & 5.7 & 9.2 & 15.4 & 12.8 \\
\hline \multirow[t]{3}{*}{ Repeat claims (before 1994) } & LT (10 122) & $0.65(3.7)$ & 28.1 & 34.5 & 36.2 & 40.4 & 44.2 \\
\hline & NLT (13 076) & $0.40(2.0)$ & 20.0 & 26.6 & 28.8 & 33.0 & 35.8 \\
\hline & NI $\left(\begin{array}{lll}10 & 122\end{array}\right)$ & $0.43(3.0)$ & 10.8 & 14.9 & 17.6 & 28.9 & 29.9 \\
\hline \multirow[t]{3}{*}{ Repeat claims (after 1994) } & LT (8355) & $0.40(2.7)$ & 15.4 & 28.1 & 30.6 & 34.2 & 41.1 \\
\hline & NLT $(10650)$ & $0.44(2.4)$ & 7.9 & 15.5 & 20.9 & 22.4 & 32.9 \\
\hline & $\mathrm{NI}(8355)$ & $0.58(4.2)$ & 9.6 & 14.2 & 5.9 & 16.0 & 17.7 \\
\hline \multirow[t]{3}{*}{ Repeat claims (before and after 1994) } & LT (23 262) & $0.43(2.6)$ & 14.1 & 25.6 & 31.3 & 37.4 & 40.1 \\
\hline & NLT (24 483) & $0.38(2.3)$ & 14.3 & 23.9 & 31.5 & 35.3 & 38.9 \\
\hline & NI (23 262) & $0.45(3.2)$ & 9.2 & 14.6 & 23.0 & 28.3 & 29.0 \\
\hline \multicolumn{8}{|l|}{ Time off work } \\
\hline \multirow{2}{*}{$<12$ weeks } & LT (44 418) & $0.41(2.6)$ & 20.0 & 29.5 & 33.3 & 37.9 & 42.8 \\
\hline & NI $(44418)$ & $0.49(3.5)$ & 7.9 & 12.3 & 16.0 & 23.1 & 23.3 \\
\hline \multirow[t]{2}{*}{$\geqslant 12$ weeks } & LT (5154) & $0.55(3.0)$ & 15.8 & 28.7 & 33.8 & 37.4 & 41.2 \\
\hline & NI (5154) & $0.50(3.1)$ & 13.5 & 16.4 & 20.9 & 28.8 & 30.7 \\
\hline \multicolumn{8}{|l|}{ Nature of injury } \\
\hline \multirow[t]{2}{*}{ Acute } & LT (44 856) & $0.41(2.7)$ & 19.9 & 29.1 & 34.4 & 38.9 & 43.1 \\
\hline & NI (44 856) & $0.49(3.5)$ & 0.81 & 12.7 & 17.2 & 25.0 & 25.4 \\
\hline \multirow[t]{2}{*}{ Chronic } & LT (4315) & $0.47(2.4)$ & 17.0 & 32.9 & 25.1 & 30.1 & 37.8 \\
\hline & NI (4315) & $0.45(3.5)$ & 9.2 & 14.0 & 13.9 & 16.4 & 17.2 \\
\hline \multicolumn{8}{|l|}{$\begin{array}{l}\text { Working entire study period (last two } \\
\text { digits of } M S P=00+\text { ) }\end{array}$} \\
\hline & LT (34 793) & $0.42(2.8)$ & 19.0 & 28.9 & 33.9 & 38.7 & 43.6 \\
\hline & NLT (45 837) & $0.38(2.3)$ & 16.7 & 23.6 & 26.8 & 32.3 & 38.1 \\
\hline & $\mathrm{NI}(34$ 793) & $0.55(0.8)$ & 7.1 & 11.2 & 13.8 & 22.7 & 23.6 \\
\hline \multicolumn{8}{|l|}{ Registered entire study period } \\
\hline & LT (39 822) & $0.43(2.7)$ & 18.5 & 28.5 & 30.7 & 35.8 & 40.3 \\
\hline & NLT $(59520)$ & $0.42(2.4)$ & 15.2 & 21.0 & 25.3 & 28.4 & 33.7 \\
\hline & $\mathrm{NI}(39822)$ & $0.51(3.6)$ & 7.2 & 10.6 & 14.2 & 20.7 & 21.9 \\
\hline
\end{tabular}

LT, lost time injured workers; NLT, no lost time injured workers; NI, non-injured comparisons.

*Totals do not add up due to missing information.

†See table 1 footnotes for details.

and a sustained decrease $(19 \%)$ by the end of the study period.

Table 2 shows the relative percentage change in mean hospital days following a workplace injury by worker claim

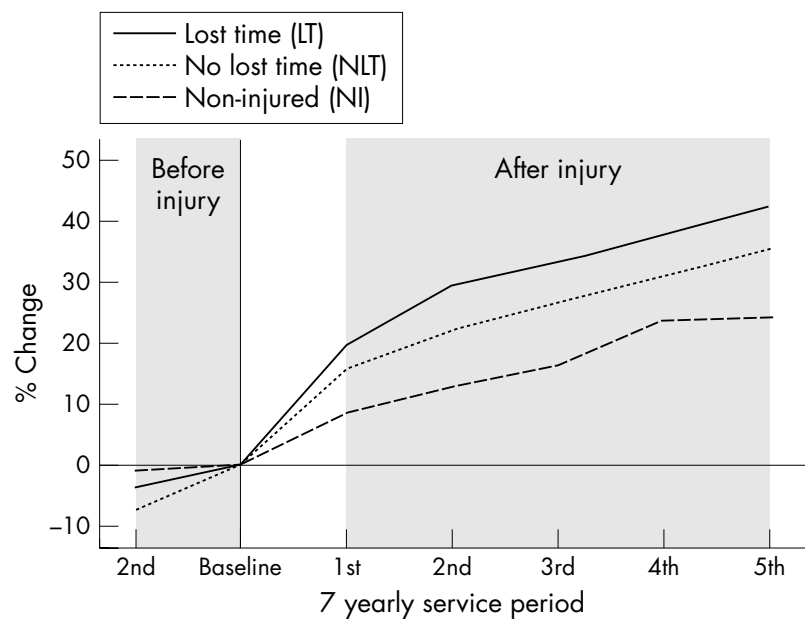

Figure 3 Mental healthcare of injured workers received from physicians five years before and five years after a 1994 workplace injury. The baseline period (vertical line) is the first yearly period immediately before the injury (see table 3). Data for other periods show percentage differences from these means. and registration characteristics. All LT repeat claimants had higher increases compared to LTs with one claim in 1994. However, even among single claimants the pattern of increase for the LTs was greater than the other two groups. LT workers who were off more than 12 weeks had a higher percentage increase in use compared to LTs who had less than 12 weeks off work. Acute LT workers had a slightly higher percentage increase in use compared to LTs with chronic injuries. In both cases, LTs had the greatest percentage increase compared to the NIs (see table 2).

When we look at individuals who were working the entire study period (last two digits of MSP number $=00$ ) and workers who were registered the entire period of the study, the pattern of LTs having the greatest increase in service use, and the NIs having the smallest increase in use, remained the same (see table 2 ).

\section{Mental healthcare use}

Twelve per cent of the LT group increased physician visits for mental healthcare one year after the injury, compared to $10 \%$ of the NLTs and $8 \%$ of the NIs. Five years after the injury, $14 \%$ of LTs had an increase in use, compared to $12 \%$ of the NLTs and $10 \%$ of the NIs (data not shown).

Figure 3 shows the percentage change in mean physician visits for mental healthcare, before and after the injury, relative to each group's mean in the year before the injury. The LT workers had the largest change in mean visits, with a steady increase to $43 \%$ by the end of the study period. Use by the other groups increased more slowly, with the NLT 
workers having a $36 \%$ increase in visits and the NI having $24 \%$ increase by the end of the study period $(p \leqslant 0.05)$. One year before the injury, the NI had on average more visits for mental illness (see table 3).

Table 3 shows the relative percentage change in mean physician visits for mental healthcare following a workplace injury by worker claim and registration characteristics. All claimants (single and repeat) had higher percentage increases, with single claimants having a slightly higher increase compared to repeat claimants. LT workers who were off more than 12 weeks had a slightly lower percentage increase in use compared to LTs who had less than 12 weeks off work. Acute LT workers had a slightly higher percentage increase in use compared to LTs with chronic injuries. In both cases, LTs had the greatest percentage increase compared to the NIs (see table 3 ).

When we look at individuals who were working the entire study period (last two digits of MSP number $=00$ ) and workers who were registered the entire period of the study, the pattern of LTs having the greatest increase in service use, and the NI having the smallest increase in use, remains the same (see table 3).

Similar patterns were seen for physician services for depression; with a steady increase to $54 \%$ more visits by the end of the study period. The other groups increased their use more slowly; with the NLT workers having a $43 \%$ increase in visits and the NI $31 \%$ increase in visits by the end of the study period $(\mathrm{p} \leqslant 0.01)$ (data not shown).

One per cent of all groups increased hospital days for mental healthcare one year after the injury. Five years after the injury, the increase was the same (data not shown).

Figure 4 shows the percentage changes in mean hospital days for mental healthcare, before and after the injury, relative to each group's mean in the year before the injury. The LT injured workers increased use by $56 \%$ on average in the year after the injury and $70 \%$ five years later. The NLT workers increased mean days of use steadily up to five years after the injury, when they reached a 55\% increase in days. In contrast, the NIs peaked at four year after the injury $(52 \%)$ $(\mathrm{p}=0.68)$.

Table 4 shows the relative percentage change in mean hospital days for mental healthcare following a workplace injury by worker claim and registration characteristics. All claimants (single and repeat) had higher percentage

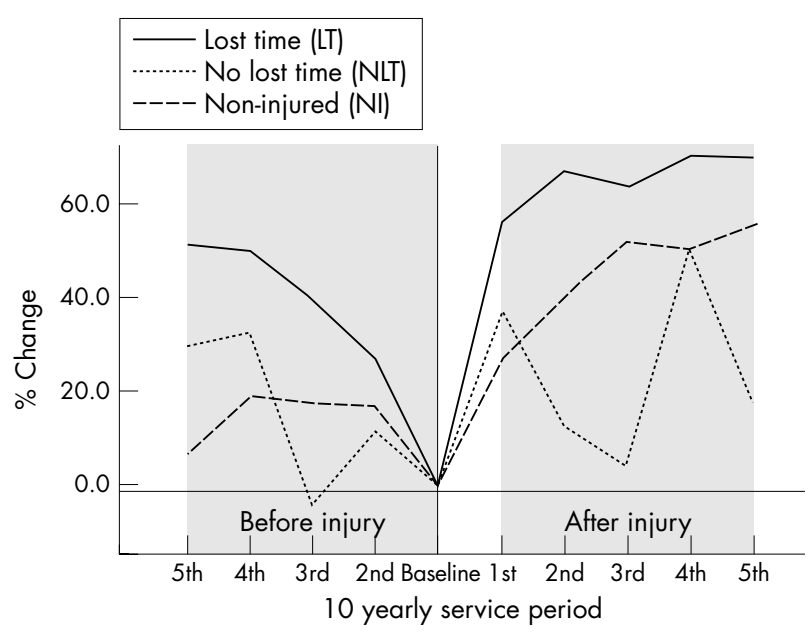

Figure 4 Mental health care of injured workers received from hospitals five years before and five years after a 1994 workplace injury. The baseline period (vertical line) is the first yearly period immediately before the injury (see table 4). Data for other periods show absolute differences from these means. increases, with single claimants having a slightly higher increase compared to repeat claimants. LT workers who were off more than 12 weeks had a slightly higher percentage increase in use compared to LTs who had less than 12 weeks off work. Acute LT workers had a slightly higher percentage increase in use compared to LTs with chronic injuries. In both cases, LTs had the greatest percentage increase compared to the NIs ( see table 4).

When we look at individuals who were working the entire study period (last two digits of MSP number $=00$ ) and workers who were registered the entire period of the study, the pattern of LTs having the greatest increase in days use, and the NI having the smallest increase in use, remains the same (see table 4).

Similar patterns were seen for hospital days for depression, with a steady increase to $75 \%$ more days by the end of the study period. The other groups increased their use more slowly, with the NLT workers having 5\% more days and the NI $21 \%$ increase in days by the end of the study period $(p=0.896)$ Before the injury, the NIs had more days in hospital for mental health care and depression (data not shown).

Thirty two per cent of the NIs with hospitalisation for mental health services were diagnosed with a psychotic condition at least once between 1989 and 1999, compared to $24 \%$ of the LT workers and $25 \%$ of the NLT workers $\left(\chi^{2}=58.7, \mathrm{df}=2 \mathrm{p} \leqslant 0.001\right)$.

Three hundred and eighty six workers filed a mental health claim in that time period and 602 were missing information on WCB injury type. The same patterns and average use for the three groups were observed, which is not surprising given the small number of mental health claims.

\section{DISCUSSION}

Our study provides clear evidence of increased healthcare use following a workplace injury. This is a population based study and about $90 \%$ of the paid workforce in BC is covered by the WCB system. ${ }^{9}$ The high linkage rate for WCB records (97\%) to healthcare services covered by $\mathrm{BC}^{\prime}$ 's universal access to the Medicare system is noteworthy, so we have virtually complete information. In essence, this study has covered an entire population of injured workers in a Canadian province, a unique dataset in the North American context. As the broad principles of workers' compensation are comparable across the continent, we believe our results to be generalisable to injured workers in Canada and the US.

In general, LT workers slightly decreased their use of GP services before a workplace injury and then increased their use steadily thereafter, compared to the NIs whose use remained relatively stable before 1994 (year of injury) and then increased only slightly thereafter. For the NLT workers, the level of increased use following the injury was between that of these two groups, although they increased GP use before the injury. These patterns persisted when adjusting for registration in the $\mathrm{BC}$ MSP and several workplace characteristics. While the NIs spent more days in hospital and averaged more mental healthcare services than the two injured worker groups, the latter had larger increases in their use of these services following the injury than the NI group. The NI actually decreased the number of days in hospital following the injury.

Hertzman et al ${ }^{9}$ used the BCLHD to examine the healthcare use of all BC workers injured in 1991. Our initial results were similar; namely for the LT injured workers, the proportion visiting their GP steadily increased from five years before the injury, peaked one year after the injury date, decreased slightly, and remained stable thereafter. The same pattern of change was observed for the NI group and the NLT injured workers, although at a lower level throughout. However, once 
Table 4 Percentage change in mean hospital days for mental healthcare following a workplace injury relative to baseline (one year before injury), by claim and registration characteristics

\begin{tabular}{|c|c|c|c|c|c|c|c|}
\hline & \multirow[b]{2}{*}{ Group $\left(n^{*}\right)$} & \multirow{2}{*}{$\begin{array}{l}\text { Baseline mean } \\
\text { days (SD) }\end{array}$} & \multicolumn{5}{|c|}{$\%$ Change following injury } \\
\hline & & & 1 year after & 2 years after & 3 years after & 4 years after & 5 years after \\
\hline \multicolumn{8}{|l|}{ Total hospital days } \\
\hline & LT (52 319) & $0.04(0.89)$ & 55.9 & 66.9 & 63.9 & 70.4 & 69.7 \\
\hline & NLT (69 142) & $0.05(2.0)$ & 26.6 & 40.5 & 52.0 & 50.5 & 55.4 \\
\hline & NI $(52319)$ & $0.14(3.8)$ & 36.4 & 12.6 & 3.9 & 51.5 & 18.1 \\
\hline \multicolumn{8}{|l|}{ Claim pattern } \\
\hline \multirow{3}{*}{ Single claim (1994 claim only) } & LT (8495) & $0.03(0.74)$ & 75.2 & 82.5 & 74.9 & 74.7 & 83.9 \\
\hline & NLT (16 565) & $0.09(3.7)$ & 15.6 & 30.6 & 47.4 & 55.5 & 36.9 \\
\hline & NI $(8495)$ & $0.12(2.0)$ & 72.3 & 36.4 & 40.7 & 4.8 & 35.0 \\
\hline \multirow[t]{3}{*}{ Repeat claims (before 1994) } & LT (10 122) & $0.05(1.1)$ & 70.9 & 73.9 & 68.3 & 80.4 & 70.5 \\
\hline & NLT (13 076) & 0.0510 .80 & 51.2 & 55.2 & 67.1 & 36.9 & 53.2 \\
\hline & NI $\left(\begin{array}{lll}10 & 122\end{array}\right)$ & $0.21(7.1)$ & 20.2 & -52.5 & -8.8 & -38.7 & -45.4 \\
\hline \multirow[t]{3}{*}{ Repeat claims (after 1994) } & LT (8355) & $0.03(0.92)$ & 30.5 & 68.9 & 66.6 & 54.4 & 67.8 \\
\hline & NLT (10 650) & $0.03(0.59)$ & 20.1 & 55.3 & 69.8 & 32.8 & 75.0 \\
\hline & NI (8355) & $0.18(5.1)$ & -107.1 & -27.3 & -90.3 & -65.6 & -50.1 \\
\hline \multirow[t]{3}{*}{ Repeat claims (before and after 1994) } & LT (23 262) & $0.04(0.83)$ & 29.8 & 44.9 & 54.3 & 63.6 & 61.4 \\
\hline & NLT (24 483) & $0.04(0.97)$ & 17.9 & 34.6 & 29.3 & 32.8 & 63.5 \\
\hline & NI $(23262)$ & $0.09(1.9)$ & 39.7 & 40.8 & 17.4 & -32.1 & 50.2 \\
\hline \multicolumn{8}{|l|}{ Time off work } \\
\hline \multirow[t]{2}{*}{$<12$ weeks } & LT (44 418) & $0.03(0.85)$ & 57.7 & 64.6 & 63.6 & 69.6 & 68.5 \\
\hline & NI $(44418)$ & $0.14(4.3)$ & 13.5 & 7.2 & -12.7 & -47.7 & 13.6 \\
\hline \multirow[t]{2}{*}{$\geqslant 12$ weeks } & LT (5154) & $0.04(0.88)$ & 47.8 & 65.0 & 73.2 & 63.0 & 77.0 \\
\hline & NI (5154) & $0.13(2.2)$ & 29.2 & 31.1 & 46.6 & -7.4 & 6.5 \\
\hline \multicolumn{8}{|l|}{ Nature of injury } \\
\hline \multirow[t]{2}{*}{ Acute } & LT (44 856) & $0.04(0.85)$ & 56.2 & 62.7 & 63.5 & 69.8 & 70.1 \\
\hline & NI (44 856) & $0.14(4.3)$ & 16.6 & 4.5 & -15.8 & -24.8 & -13.3 \\
\hline \multirow[t]{2}{*}{ Chronic } & LT (4315) & $0.04(0.97)$ & 59.4 & 83.8 & 54.8 & 80.8 & 68.3 \\
\hline \multirow{2}{*}{\multicolumn{8}{|c|}{$\begin{array}{l}\text { Working entire study period (last two } \\
\text { digits of } M S P=00+\text { ) }\end{array}$}} \\
\hline & & & & & & & \\
\hline & LT (34 793) & $0.04(0.95)$ & 46.6 & 67.8 & 64.6 & 73.5 & 71.6 \\
\hline & NLT ( 45 837) & $0.05(2.3)$ & 17.1 & 37.5 & 51.7 & 54.8 & 58.8 \\
\hline & NI (34 793) & $0.14(2.8)$ & 50.0 & 25.6 & 4.7 & -13.5 & 28.7 \\
\hline \multicolumn{8}{|l|}{ Registered entire study period } \\
\hline & LT (39 822) & $0.03(0.85)$ & 52.3 & 69.2 & 64.5 & 71.6 & 70.2 \\
\hline & NLT (59 520) & $0.05(2.0)$ & 23.9 & 42.4 & 49.5 & 44.9 & 51.6 \\
\hline & NI (39 822) & $0.14(4.3)$ & 29.4 & 2.1 & 1.5 & -11.2 & 11.8 \\
\hline
\end{tabular}

we adjusted for registration in the BC MSP, all groups showed constant use before the injury and both worker groups increased use more than the NI following the injury. Given the data limitations at the time, Hertzman et al ${ }^{9}$ were unable to control for registration in the BC MSP.

Our comparison group was selected using Hertzman et al's ${ }^{9}$ criteria-workers in the general population who were registered for health benefits but did not submit a WCB claim during the full study period (1989-99). This no doubt resulted in a group at lower risk for a workplace injury than the injured worker groups. In particular, this group likely overrepresents people with chronic disabilities who were unable to work, and used fewer GP services but more hospital services. The fact that the NI group tended to spend more days in hospital and were more likely to be diagnosed with a psychotic form of mental illness reinforces this hypothesis. The higher mean visits for the NI group may be because a proportion of them had a more serious mental illness diagnosis and more serious diagnoses, in general. However, use was examined for the workers five years before the injury and the injured workers clearly showed a change in use. Our group of NI workers was intended to identify trends rather than absolute levels of service use, and so is not considered a control group, in the traditional sense. However, we believed it could still be used to show secular trends in healthcare use. In any event, we have done an analysis restricted to those whose last two digits of the MSP were 00 throughout the study period (that is, individuals employed the entire time) and our results were essentially the same.
Unfortunately we could not eliminate healthcare services covered by the WCB from the data set. This may account for some of the increase in physician use in the year after injury for the workers. However, we were able to control for WCB related healthcare use in the hospital separation data and found a greater change in use after the injury for both injured worker groups than for the comparison group. We also performed a subanalysis excluding injured workers who filed a WCB claim for mental health services (a very small number) and found a greater increase in mental healthcare use for both groups of injured workers compared with the comparison group.

The modest increases (although significant) in mental healthcare among injured workers is inconsistent with recent qualitative evidence documenting the consequences of workplace injuries, and the resulting time off work, on the emotional wellbeing and quality of life of injured workers. For example, feelings of depression and anxiety have been reported in US workers with repetitive strain disorder. ${ }^{14}$ Injured workers in an ethnographic study who lost more than 28 days from work described feelings of depression and anger, and stress and strain on family relationships because of the limitations due to their chronic back pain injury. ${ }^{8}$ Similarly, hospital workers in Ohio said that they experienced depression and anxiety due to their injury, ${ }^{8}$ while injured workers in Pennsylvania reported sleep disturbances, lowered self-esteem, and occasional suicidal tendencies. ${ }^{15}$ Perhaps severe effects in a small proportion of people are diluted at the population level. 


\section{Main messages}

- Lost time injured workers increased physician visits, hospital days, and mental healthcare use, five years after the injury, relative to the year before the injury, at a higher rate than the non-injured comparison group.

- For the no lost time injured workers, the level of increased use following the injury was between that of these two groups.

- These patterns persisted when adjusting for registration in the BC Medical Service Plan (MSP) and several workplace characteristics.

\section{Policy implications}

- Any increase in use among injured workers that is directly or indirectly related to the injury should be paid for by the WCB system, which is funded by employer premiums.

- If it is paid by MSP, this represents a cost shift, in the Canadian context, from the private to the public sector - an important issue, especially at a time when the healthcare systems in both Canada and the US are under financial strain.

Our analysis examined mental healthcare services provided by all physician types (the majority of whom are GPs). According to a recent report from the British Columbia Psychological Association, ${ }^{16}$ in primary care physicians' offices, nearly $50 \%$ of cases of depression and anxiety go unreported and around one third are misdiagnosed. When we restricted our analysis to services provided by psychiatrists only, we found very little difference among the three groups. Regardless, we would not expect differential bias among GPs for reporting mental healthcare use for the three groups.

Our findings of increased overall healthcare use following a workplace injury have important public policy implication. For example, any increase in use among injured workers that is directly or indirectly related to the injury should be paid for by the WCB system, which is funded by employer premiums. If it is paid by the MSP, this represents a cost shift, in the Canadian context, from the private to the public sector-an important issue, especially at a time when the healthcare systems in both Canada and the US are under financial strain.

In conclusion, the linked data provided an opportunity to examine the health service use of workers before and after a workplace injury. Although the WCB system is the primary mechanism for processing claims and providing information about workplace injury, it is clear that the consequences of workplace injury extend beyond what is covered by the WCB into the publicly funded healthcare system. The increased overall healthcare use following an injury suggests the need for policy makers to focus not only on prevention but also on the social and economic consequences after a workplace injury.

\section{ACKNOWLEDGEMENTS}

The authors would like to thank the Centre for Health Services and Policy Research (CHSPR) for developing the databases and the British Columbia (BC) Ministry of Health and the BC Workers' Compensation Board for allowing us to use the data. Thank you to the National Institute for Occupational Safety and Health (NIOSH) for funding this research.

\section{Authors' affiliations}

J A Brown, University of Toronto, Toronto, ON, Canada, McMaster University, Hamilton, ON, Canada

P McDonough, University of Toronto, Toronto, ON, Canada

C A Mustard, University of Toronto, Toronto, ON, Canada, Institute of Work and Health, Toronto, ON, Canada

H S Shannon, McMaster University, Hamilton, ON, Canada, Institute for Work and Health, Toronto, ON, Canada

Competing interests: none.

\section{REFERENCES}

1 Association of Workers' Compensation Boards of Canada. Work Injuries and Diseases. Number of Accepted Time-loss Injuries, by Province, 19822003. National Work Injuries Statistics Program 2005;Available at http:// www.awcbc.org/english/NWISP_Stats.htm (accessed March 2006).

2 Bureau of Labor Statistics (BLS). Workplace injuries and illnesses in 2003, United States Department of Labor, 2005:1-16.

3 National Institue for Occupational Safety and Health. National Occupational Research Agenda (NORA) update 2001. NIOSH 2001; Pub No 2001-147, $1-48$.

4 Pransky G, Benjamin K, Hill-Fotouhi C, et al. Outcomes in work-related upper extremity and low back injuries: results of a retrospective study. Am J Ind Med 2000;37:400-9.

5 Keller SD. Quantifying social consequences of occupational injuries and illnesses: state of art and research agenda. Am J Ind Med 2001;40:438-51.

6 Adams M, Burton J, Butcher F, et al. Aftermath: the social and economic consequences of workplace injury and illness. New Zealand Department of Labour, 2002:1-246.

7 Kirch B, Mckee P. The needs and experiences of injured workers: a participatory research study. Work 2003;21:221-31.

8 Strunin L, Boden L. Family consequences of chronic back pain. Soc Sci Med 2004;58:1385-93.

9 Hertzman C, McGrail K, Hirtle B. Overall pattern of health care and social welfare use by injured workers in the British Columbia Cohort. Int J Law Psychiatry 1999;22:581-601.

10 WorkSafe BC. Workers' Compensation Board of British Columbia 2005 Available at http://search.worksafebc.com (accessed March 2006).

11 Menard M. Comparison of disability of behavior after different sites and types of injury in a workers' compensation population. J Occup Environ Med 1996;38:1161-70

12 Martens P. Patterns of regional mental illness disorder diagnoses and service use in Manitoba: a population-based study, Manitoba Centre for Health Policy, University of Manitoba, 2004.

13 Tataryn D, Mustard C, Derksen S. The utilization of medical services for mental health disorders Manitoba: 1991-1992. Manitoba Centre for Health Policy, Report \#2356, 1994.

14 Morse TF, Dillon C, Warren N, et al. The economic and social consequences of work-related musculoskeletal disorders: the Connecticut Upper-extremity Surveillance Project (CUSP). Int J Occup Environ Health 1998;4:209-16.

15 Dawson SE. Workers' Compensation in Pennsylvania: the effects of delayed contested cases. J Health Soc Policy 1994;6:87-100.

16 Lea G, Crawford T. An expanded role for psychology in British Columbia's mental and behavioural health care. British Columbia Psychology Association, 2003: 1-18. 